\title{
Introduction to Volume 1
}

In the first volume of this book, we give introductory accounts of topological dynamical systems acting on compact metrizable spaces, of finite-alphabet symbolic systems, and of ergodic theory of measure-theoretic dynamical systems acting on probability spaces, the latter including the metric entropy theory of Kolmogorov and Sinai. More advanced topics include infinite ergodic theory, general thermodynamic formalism, and topological entropy and pressure. This volume also includes a treatment of several classes of dynamical systems, which are interesting on their own and will be studied at greater length in the second volume: we provide a fairly detailed account of distance expanding maps and discuss Shub expanding endomorphisms, positively expansive maps, and homeomorphisms and diffeomorphisms of the circle.

We now describe the content of each chapter of this first volume in more detail, including their mutual dependence and interrelations.

\section{Chapter 1 - Dynamical systems}

In the first few sections of Chapter 1, we introduce the basic concepts in the theory of topological dynamical systems: orbits, periodic points, preperiodic points, $\omega$-limit sets, factors, and subsystems. In particular, we introduce the concept of topological conjugacy and identify the number of periodic points of any given period as a simple (topological conjugacy) invariant. We further examine the following invariants: minimality, transitivity, topological mixing, strong transitivity, and topological exactness. Finally, we provide the first two classes of examples, namely rotations on compact topological groups and some continuous maps on compact intervals.

\section{Chapter 2 - Homeomorphisms of the circle}

In Chapter 2, we temporarily step away from the general theory of dynamical systems to consider more specific examples. We investigate homeomorphisms of the unit circle and examine the notions of lift and rotation number for homeomorphisms. Then we study in more detail the subclass of diffeomorphisms of the unit circle. The main result of this chapter is Denjoy's theorem, which states that if a $C^{2}$ diffeomorphism has an irrational rotation number, then this diffeomorphism is a minimal system which is topologically conjugate to an irrational rotation.

\section{Chapter 3 - Symbolic dynamics}

In Chapter 3, we discuss symbolic dynamical systems. We treat them as objects in their own right, but later (in Chapter 4, among others) we apply the ideas developed here to more general systems. We restrict ourselves to the case of finitely many letters, as symbolic systems born out of finite alphabets give rise to systems acting on compact metrizable spaces. Nevertheless, note that in Chapter 17 of the second volume, 
we will consider countable-alphabet symbolic dynamics. In Section 3.1, we introduce full shifts. In Section 3.2, we study subshifts of finite type and in particular the characterizations of topological transitivity and exactness in terms of the underlying matrix associated with such systems. Finally, in Section 3.3 we examine general subshifts of finite type.

\section{Chapter 4 - Distance expanding maps}

In Chapter 4, we define and give some examples of distance expanding maps. In Section 4.2, we study the properties of their local inverse branches. This is a way of dealing with the noninvertibility of these maps. In Section 4.3, we examine the all important concepts of pseudo-orbit and shadowing. In Section 4.4, we introduce the powerful concept of Markov partitions and establish their existence for open, distance expanding systems. We then show in Section 4.5 how to use Markov partitions to represent symbolically the dynamics of open, distance expanding systems. This is a beautiful application of the symbolic dynamics studied in Chapter 3. The final theorem of the chapter describes the properties of the coding map between the underlying compact metric space (the phase space) and some subshift of finite type (a symbolic space).

\section{Chapter 5 - Expansive maps}

In Chapter 5, we introduce the concept of expansiveness. Amidst the large variety of dynamical behaviors, which can be thought of as expansionary in some sense, expansiveness has turned out to be a rather weak but useful notion. Indeed, all distance expanding maps are expansive and so, more particularly, all subshifts over a finite alphabet are expansive. But expansiveness is not so far from expandingness, as we demonstrate in this chapter that every expansive system is in fact expanding with respect to some metric compatible with the topology. This means that many of the results proved in Chapter 4, such as the existence of Markov partitions and of a nice symbolic representation, the density of periodic points, the closing lemma, and the shadowing property, hold for all positively expansive maps. Nevertheless, expansiveness is weaker than expandingness, and we provide at the end of the chapter a class of expansive maps that are not distance expanding. Expansive maps are important for other reasons as well. One of them is that expansiveness is a topological conjugacy invariant. More crucially, the measure-theoretic entropy function is upper semicontinuous within that class of maps. In particular, all expansive maps admit a measure of maximal entropy and, more generally, equilibrium states under all continuous potentials (see Chapter 12).

\section{Chapter 6 - Shub expanding endomorphisms}

In Section 6.2, we give a systematic account of Shub's expanding endomorphisms. These maps constitute a large, beautiful subclass of distance expanding maps and 
are far-reaching generalizations of the expanding endomorphisms of the circle, which will be first introduced in Section 6.1. After a digression into albegraic topology, we establish in Section 6.4 that Shub expanding endomorphisms are structurally stable, form an open set in an appropriate topology of smooth maps, are topologically exact, have at least one fixed point as well as a dense set of periodic points, and their universal covering space is diffeomorphic to $\mathbb{R}^{n}$.

\section{Chapter 7 - Topological entropy}

In Chapter 7, we study the central notion of topological entropy, one of the most useful and widely-applicable topological invariant thus far discovered. It was introduced to dynamical systems by Adler, Konheim, and McAndrew in 1965. Their definition was motivated by Kolmogorov and Sinai's definition of metric/measure-theoretic entropy introduced less than a decade earlier. The topological entropy of a dynamical system, which we introduce in Section 7.2, is a nonnegative extended real number that measures the complexity of the system. Topological entropy is a topological conjugacy invariant but by no means a complete invariant. In Section 7.3, we treat at length Bowen's characterization of topological entropy in terms of separated and spanning sets. In Chapter 11, we will introduce and deal with topological pressure, which is a substantial generalization of topological entropy. Our approach to topological pressure will stem from and extend that for topological entropy. In this sense, this chapter can be viewed as a preparation to Chapter 11 .

\section{Chapter 8 - Ergodic theory}

In Chapter 8, we move away from the study of purely topological dynamical systems to consider instead dynamical systems that come equipped with a measure. That is, instead of self-maps acting on compact metrizable spaces, we now ask that the selfmaps act upon measure spaces. We introduce in Section 8.1 the basic object of study in ergodic theory, namely, invariant measures. We also prove Poincaré's recurrence theorem. Section 8.2 presents the notion of ergodicity and comprises a demonstration of Birkhoff's ergodic theorem. This theorem is one of the most fundamental results in ergodic theory. It is extremely useful in numerous applications. The class of ergodic measures for a given transformation is then studied in more detail. The penultimate Section 8.3 contains an introduction to various measure-theoretic mixing properties that a system may satisfy, and shows that ergodicity is a very weak form of mixing. In the final Section 8.4, Rokhlin's natural extension of any given dynamical system is described and the mixing properties of this extension are investigated.

\section{Chapter 9 -Measure-theoretic entropy}

In Chapter 9, we study the measure-theoretic entropy of a (probability) measurepreserving dynamical system, also known as metric entropy or Kolmogorov-Sinai 
metric entropy. It was introduced by A. Kolmogorov and Ya. Sinai in the late 1950s. Since then, its account has been presented in virtually every textbook on ergodic theory. Its introduction to dynamical systems was motivated by Ludwig Boltzmann's concept of entropy in statistical mechanics and Claude Shannon's work on information theory. We first study measurable partitions in Section 9.2. Then we examine the concepts of information and conditional information in Section 9.3. In Section 9.4, we finally define the metric entropy of a measure-preserving dynamical system. And in Section 9.5, we formulate and prove the full version of Shannon-McMillan-Breiman's characterization of metric entropy. Finally, in Section 9.6 we shed further light on the nature of entropy, by proving the Brin-Katok local entropy formula. Like the Shannon-McMillan-Breiman theorem, the Brin-Katok local entropy formula is very useful in applications.

\section{Chapter 10 - Infinite invariant measures}

In Chapter 10, we deal with measurable transformations preserving measures that are no longer assumed to be finite. The outlook is then substantially different than in the case of finite measures. In Section 10.1, we investigate in detail the notions of quasi-invariant measures, ergodicity, and conservativity. We also prove Halmos' recurrence theorem, which is a generalization of Poincaré's recurrence theorem for quasiinvariant measures that are not necessarily finite. In Section 10.2, we discuss first return times, first return maps, and induced systems. We further establish relations between invariant measures for the original transformation and the induced one. In Section 10.3, we study implications of Birkhoff's ergodic theorem for finite and infinite measure spaces. Among others, we demonstrate Hopf's ergodic theorem, which applies to measure-preserving transformations of $\sigma$-finite spaces. Finally, in Section 10.4, we seek a condition under which, given a quasi-invariant probability measure, one can construct a $\sigma$-finite invariant measure which is absolutely continuous with respect to the original measure. To this end, we introduce a class of transformations, called Martens maps, that have this feature and even more. In fact, these maps have the property that any quasi-invariant probability measure admits an equivalent $\sigma$-finite invariant one. Applications of these concepts and results can be found in Chapters 13-14 of the second volume and Chapters 29-32 of the third volume.

\section{Chapter 11 - Topological pressure}

\section{Chapter 12 - The variational principle and equilibrium states}

In the last two chapters of this first volume, we introduce and extensively deal with the fundamental concepts and results of thermodynamic formalism, including topological pressure, the variational principle, and equilibrium states. This topic has a continuation throughout the whole second volume, first and perhaps most notably, in the first chapter of that volume, which is devoted to the thermodynamic formalism of distance expanding maps and Hölder continuous potentials. It will be enriched by 
the seminal concepts of Gibbs states and transfer (Perron-Frobenius, Ruelle, Araki) operators.

Thermodynamic formalism originated in the late 1960s with the works of David Ruelle. The motivation for Ruelle came from statistical mechanics, particularly glass lattices. The foundations, classical concepts and theorems of thermodynamic formalism were developed throughout the 1970s by Ruelle, Rufus Bowen, Peter Walters, and Yakov Sinai.

In Chapter 11, we define and investigate the properties of topological pressure. Like topological entropy, this is a topological concept and a topological conjugacy invariant. We further give Bowen's characterization of pressure in terms of separated and spanning sets.

In Chapter 12, we relate topological pressure with metric entropy by proving the variational principle, the very cornerstone of thermodynamic formalism. This principle naturally leads to the concepts of equilibrium states and measures of maximal entropy. Among others, we show that under a continuous potential every expansive dynamical system admits an equilibrium state. 
